\title{
KOTO Detector Status
}

\section{Takahiko Masuda* for the КОТО collaboration}

Department of Physics, Kyoto University, Kyoto 606-8502, Japan

E-mail: taka@scphys.kyoto-u.ac.jp

The KOTO experiment at the J-PARC laboratory seeks to achieve the first observation of the $K_{L} \rightarrow \pi^{0} v \bar{v}$ decay, as a direct measurement of the CP-violating parameter in the Standard Model. Many improvements are being made over the previous KEK E391a experiment. Almost all the detector subsystems have been fabricated and installed by December 2012. This paper describes the detector performance and calibration methods based on the experience of the engineering run in January 2013.

2013 Kaon Physics International Conference,

29 April-1 May 2013

University of Michigan, Ann Arbor, Michigan - USA

\footnotetext{
* Speaker.
} 


\section{Introduction}

The CP violation phenomena observed so far in laboratories have been explained by the complex phase that naturally comes into the $3 \times 3$ quark-mixing matrix introduced by Kobayashi and Maskawa. This theory was supported by the $\Re\left(\varepsilon^{\prime} / \varepsilon\right)$ measurements at CERN and Fermilab, and by the results from the B-factory experiments at KEK and SLAC. Although this CP-violating mechanism was built into the standard model of particle physics, it still cannot explain the matterantimatter asymmetry in the universe. There should be another CP violation mechanism introduced by new physics beyond the standard model [1].

The KOTO experiment [2] at the J-PARC laboratory searches for new physics that breaks the CP symmetry. The probe that we use is the $K_{L} \rightarrow \pi^{0} v \bar{v}$ decay. Because this decay directly breaks the CP symmetry, and contains a loop in the diagrams of this decay, it is sensitive to new particles [3]. In the standard model, this decay mode is suppressed and the theoretical uncertainty in the branching ratio calculation is small. Thus, in relative terms, the new physics effect is expected to be large.

\section{Design Concepts}

The KOTO detector can be divided into two parts. One is an electromagnetic calorimeter, and the other is a group of veto counters. A detector side view is shown in Figure 1. To identify the $K_{L} \rightarrow \pi^{0} v \bar{v}$ decay, the CsI calorimeter measures the energy and position of the two photons from $\pi^{0}$ decay, and veto counters ensure that there is no extra particle from the $K_{L}$ decay.

Figure 2 shows the $\pi^{0}$ reconstruction method. Firstly we take two electromagnetic showers in the CsI calorimeter. Assuming that these are two photons coming from a $\pi^{0}$, we can calculate the
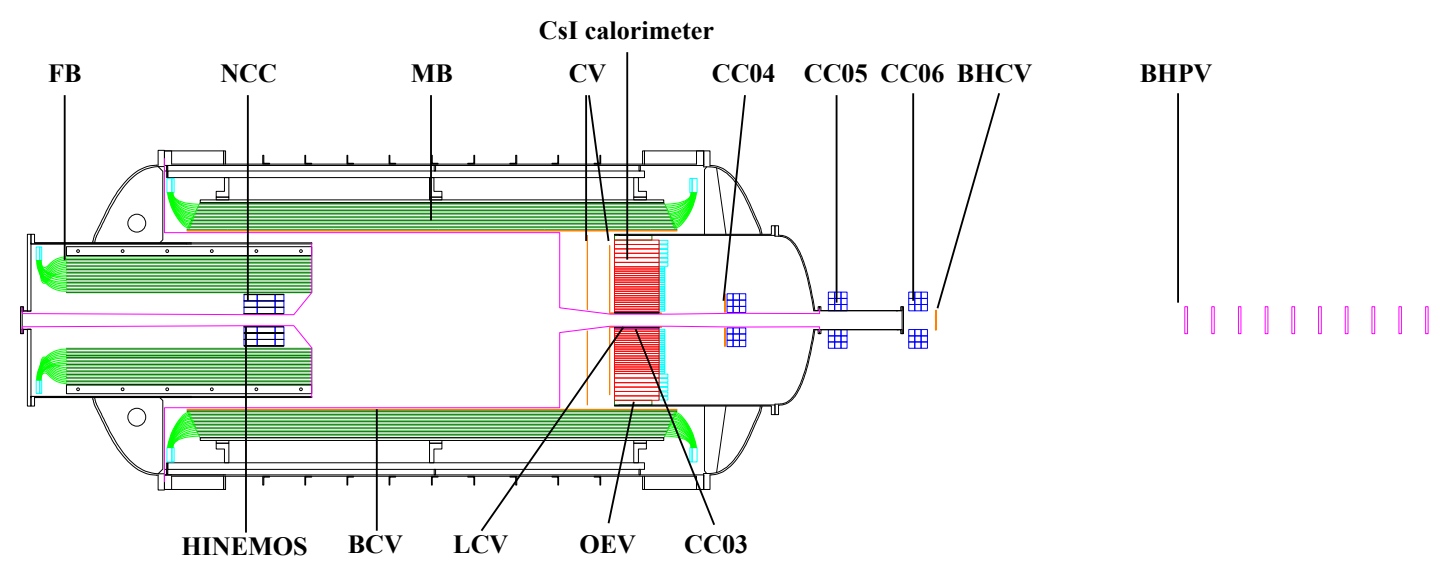

Figure 1: Side view of the KOTO detector. $K_{L}$ beam enters from the left side. HINEMOS, BCV, CV, LCV and $\mathrm{BHCV}$ are charged-particle veto counters made of thin plastic scintillators. $\mathrm{FB}$ and $\mathrm{MB}$ are photon veto counters made of lead-scintillator sandwich. NCC, CC04, CC05 and CC06 are photon veto counters made of CsI crystals. BHPV, which is located at the most downstream part, is the veto counters for the photons passing through the beam hole. Almost all the subsystems are located inside the vacuum vessel. The vacuum region surrounded by $\mathrm{MB}$ is the $K_{L}$ decay region. 
angle $\theta$ between the direction vectors of the two photons as

$$
\cos \theta=1-\frac{M_{\pi^{0}}^{2}}{2 E_{1} E_{2}}
$$

where $M_{\pi^{0}}$ is the nominal mass of $\pi^{0}$ and $E_{1}, E_{2}$ are the energies of the two photons. Assuming that the $\pi^{0}$ has decayed on the beam axis, we are able to obtain the vertex. For the $K_{L} \rightarrow \pi^{0} v \bar{v}$ decay, neutrinos carry away transverse momenta and the reconstructed $\pi^{0}$ should have a finite transverse momentum.

\subsection{CsI Calorimeter}

The CsI calorimeter reconstructs the electromagnetic showers of two photons from $\pi^{0}$ in the $K_{L} \rightarrow \pi^{0} v \bar{v}$ decay. In the KOTO experiment, the calorimeter consists of 2716 undoped-CsI crystals. We use $2.5 \times 2.5 \times 50 \mathrm{~cm}^{3}$ crystals for the central region (2240 blocks) and $5.0 \times 5.0 \times 50$ $\mathrm{cm}^{3}$ crystals for the outer region (476 blocks) of the calorimeter. The diameter is $190 \mathrm{~cm}$, and the depth is 27 radiation lengths $\left(X_{0}\right)$. The front view of the CsI calorimeter is shown in Figure 3. Each small (large) crystal is viewed from the downstream end by a 3/4 (1.5) inch Hamamatsu R5364 (R5330) phototubes.

The calorimeter was operated under stable conditions in vacuum during the engineering run (Section 3). It was calibrated with cosmic rays and, to improve on the energy calibration, with reconstructed $K_{L} \rightarrow 3 \pi^{0}$ events. The correction factors from the gain values obtained by the cosmicray based calibration were adjusted to make the width of the reconstructed $K_{L}$ mass narrower.

\subsection{Charged Veto Counters}

The Charged Veto (CV) counter identifies charged particles before entering the calorimeter. It is divided in two layers placed $5 \mathrm{~cm}$ and $30 \mathrm{~cm}$ upstream of the calorimeter front face. Each layer consists of multiple $3 \mathrm{~mm}$-thick plastic scintillator strips with wavelength shifting (WLS) fibers. The energy deposit is measured by multi-pixel photon counters at the two ends of each strip. A

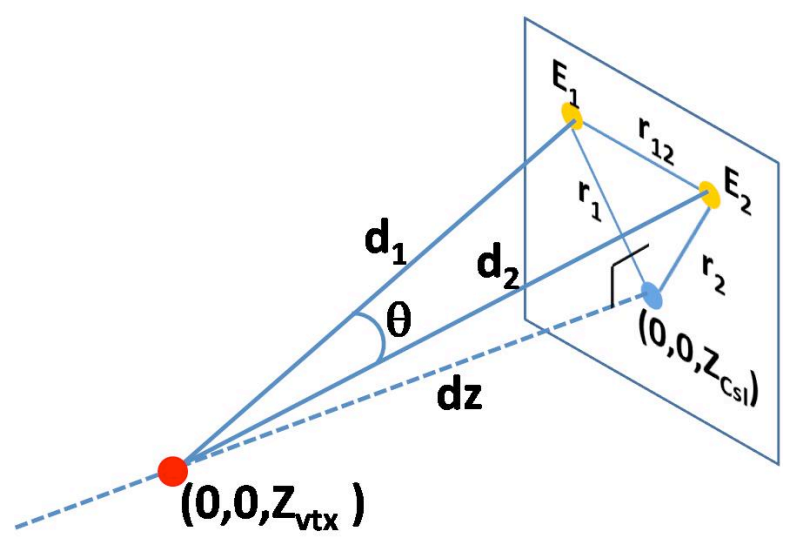

Figure 2: $\pi^{0}$ reconstruction method: the two photons from the $\pi^{0}$ decay hit the CsI calorimeter, depositing the $E_{1}$ and $E_{2}$ energy, respectively. The $\pi^{0}$ vertex is constrained along the beam axis, shown as a dashed line. 
schematic view of CV is shown in Figure 4. The light yield is more than 10 photoelectrons/100 $\mathrm{keV}$ across the whole detection region [5].

Figure 1 shows the location of the other charged-particle counters in the KOTO detector: the Barrel Charged Veto (BCV) and the Beam Hole Charged Veto (BHCV) cover the regions upstream and downstream of the calorimeter while the Liner Charged Veto (LCV) covers the beam hole inside the calorimeter.

\subsection{Photon Veto Counters}

The Main Barrel (MB) surrounds the $K_{L}$ decay region and is made of $5.5 \mathrm{~m}$ long photon veto counters. It was used in the previous E391a experiment [4]. Each module is made of a lead-scintillator sandwich. The total thickness is $14 X_{0}$. Each module is readout via WLS fibers connected to phototubes (PMT) located at both ends of the counters. Using the 2-PMTs-readout scheme, we can correct for the attenuation and propagation time of the scintillating light [6]. The KOTO detector has other photon veto counters, as shown in Figure 1: the Front Barrel (FB) located upstream of the CsI calorimeter; collar counters placed at different locations around the beam line (NCC, $\mathrm{CC} 03, \mathrm{CC} 04, \mathrm{CC} 05$ and CC06); the Outer Edge Veto (OEV) situated on the outside perimeter of the CsI calorimeter; and the Beam Hole Photon Veto (BHPV) set downstream of the CsI calorimeter.

\section{Results from the engineering run}

In December 2012 we finished the detector construction inside the vacuum vessel and, from December 2012 to January 2013, we performed an engineering run. We confirmed the stable operation of all the detector subsystems in vacuum, trigger/DAQ and slow control system. Detector calibration methods and the performance of the whole detector system were established.

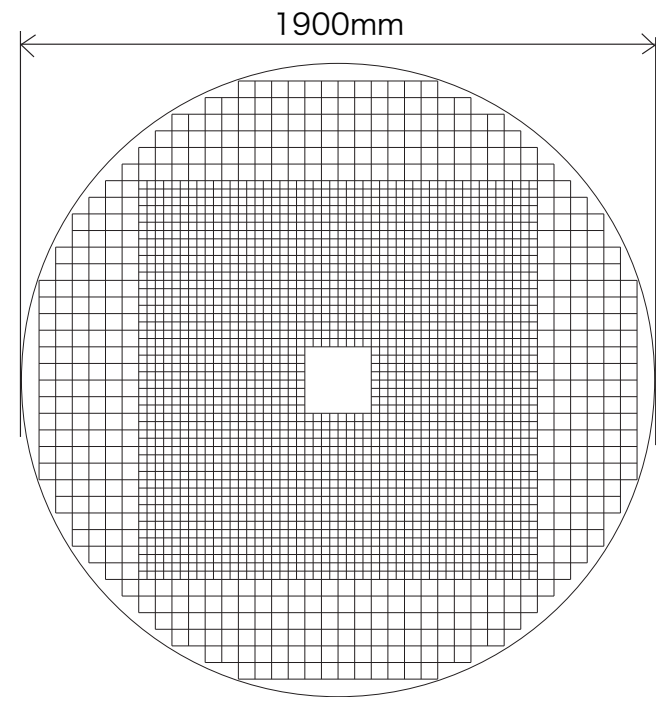

Figure 3: Front view of the CsI calorimeter.

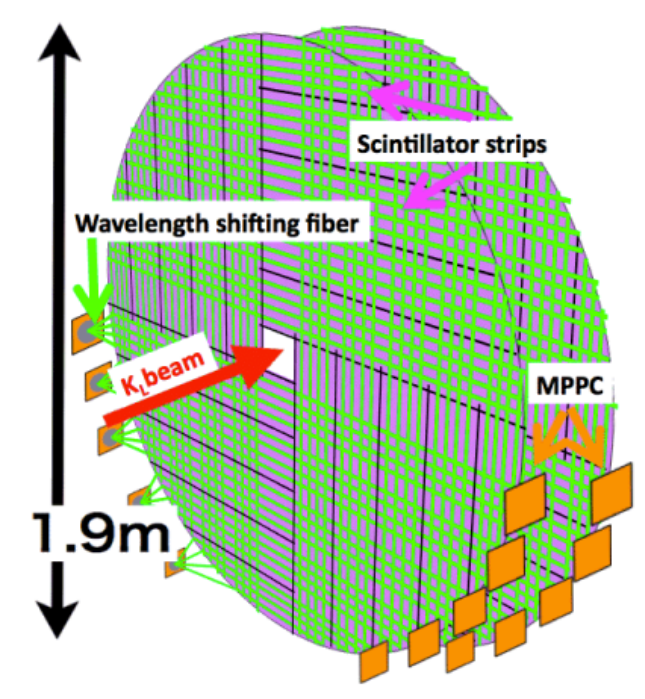

Figure 4: Schematic view of CV, which consists of two layers and is readout by WLS fibers and MPPCs. 


\subsection{Neutral decay modes}

We checked the $K_{L}$ beam yield and profile, and studied the detector performance, using $K_{L} \rightarrow$ $3 \pi^{0}$ and $K_{L} \rightarrow 2 \pi^{0}$ decays. $K_{L} \rightarrow 3 \pi^{0}$ and $K_{L} \rightarrow 2 \pi^{0}$ were reconstructed from six and four showers detected in the CsI calorimeter, respectively. In the reconstruction, the number of possible combinations of the two photon pairs is 15 for $K_{L} \rightarrow 3 \pi^{0}$ and 3 for $K_{L} \rightarrow 2 \pi^{0}$. The decay vertex position is calculated from the energy and position of the two photons of each pair by assuming the nominal $\pi^{0}$ mass. To decide on the photon assignment, the variance of the reconstructed vertex points, named "pairing $\chi_{z}^{2}$ ", was calculated for all possible combinations, and then we selected the pairing with the smallest $\chi_{z}^{2}$

In the $K_{L} \rightarrow 3 \pi^{0}$ analysis, we require the number of the showers in the calorimeter to be six. There is no other considerable process making six or more showers from $K_{L}$ decays except for the Dalitz decay from $\pi^{0}$, and thereby the calorimeter has the whole information on the original $K_{L}$ decay. Figures 5 and 6 show a schematic view and an event display of $K_{L} \rightarrow 3 \pi^{0}$, respectively. Using this decay mode, we checked the basic performance of the CsI calorimeter.

$K_{L} \rightarrow 2 \pi^{0}$ events are important for us to check the performance of the hermetic veto system. As shown in Figure 7 and Figure 8, we analyze events with four photons to the calorimeter and no photons to the veto counters. The main backgrounds are $K_{L} \rightarrow 3 \pi^{0}$ and $K_{L} \rightarrow \pi^{+} \pi^{-} \pi^{0}$ decays. Figure 9 shows a schematic view of these background events with four showers in the calorimeter. For $K_{L} \rightarrow 3 \pi^{0}$ decay, there should be two extra photons somewhere in the photon veto counters, and for $K_{L} \rightarrow \pi^{+} \pi^{-} \pi^{0}$ decay, the charged particle veto counters will record some activity.

\subsection{Preliminary results}

Plots from the engineering run are presented in this subsection. Figure 10 shows the reconstructed mass of $K_{L} \rightarrow 3 \pi^{0}$ events. The events in the tail consist of $K_{L} \rightarrow 3 \pi^{0}$ due to energy mismeasurement or mis-pairing of two photons. Figure 11 shows the reconstructed mass of $K_{L} \rightarrow 2 \pi^{0}$ events. In this analysis we imposed the veto requirements on CV and MB. The main background comes from $K_{L} \rightarrow 3 \pi^{0}$ when the two photons hit outside the calorimeter region. In this case, we

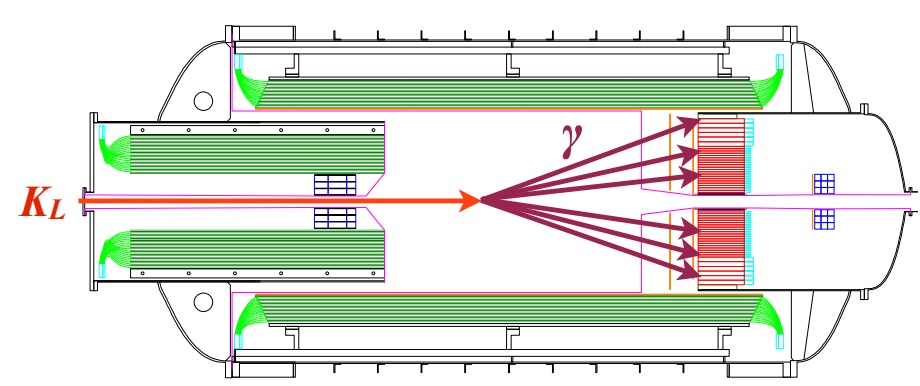

Figure 5: Side view of the detector with the $K_{L} \rightarrow 3 \pi^{0}$ decay.

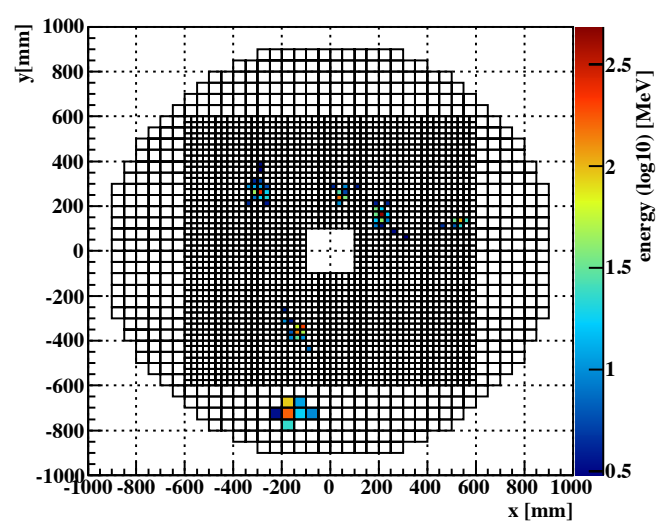

Figure 6: Example of an event display with the $K_{L} \rightarrow$ $3 \pi^{0}$ decay. 

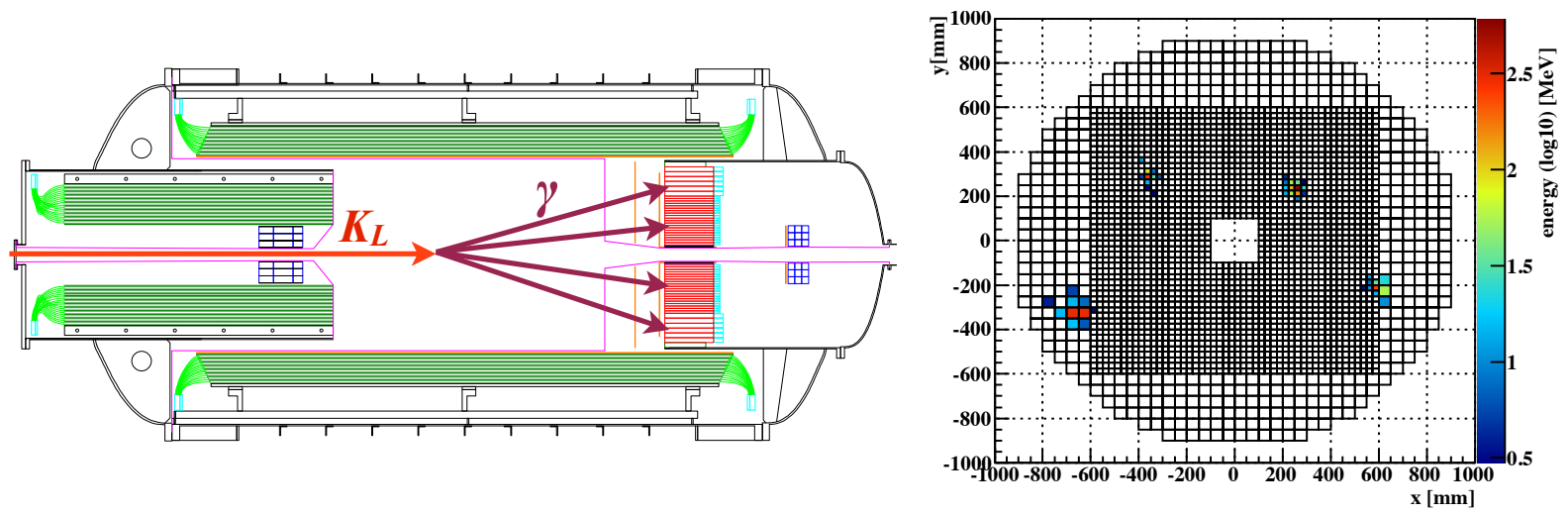

Figure 7: Side view of the detector with the $K_{L} \rightarrow 2 \pi^{0}$ decay. Figure 8: Example of an event display with the $K_{L} \rightarrow$ $2 \pi^{0}$ decay.
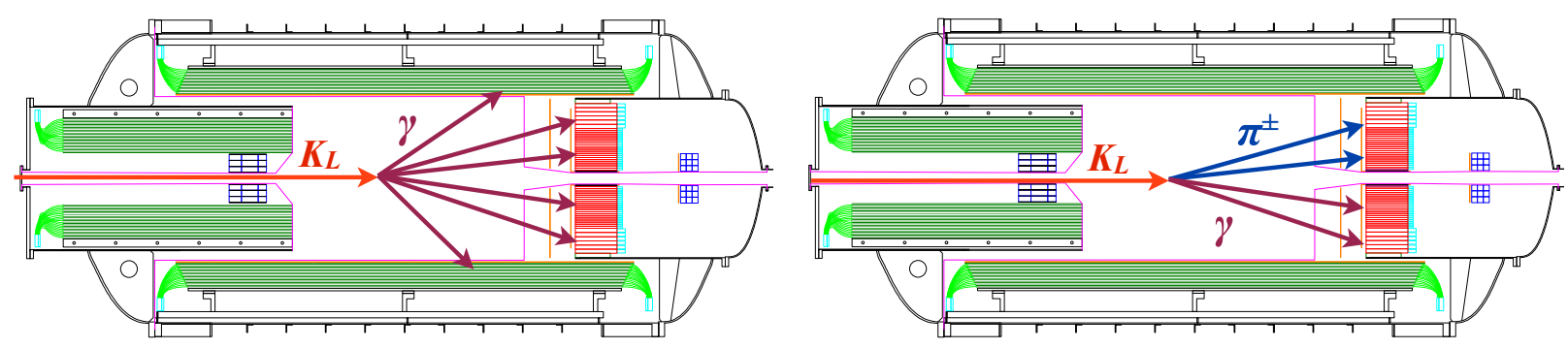

Figure 9: Side view of the background events to the $K_{L} \rightarrow 2 \pi^{0}$ event; (left) $K_{L} \rightarrow 3 \pi^{0}$ background, (right) $K_{L} \rightarrow \pi^{+} \pi^{-} \pi^{0}$ background. Both backgrounds have four showers in the calorimeter

expect some activity in the MB detector due to the extra photons. Figure 12 shows the energy deposits in MB from the $K_{L} \rightarrow 2 \pi^{0}$ analysis. The green spectrum in the figure shows the accidental activities in MB, and was measured by the off-timing hits. The blue spectrum shows the energy deposits from background events. The contribution due to accidental hits has already been subtracted. The red spectrum shows the simulated energy deposits from the background. Simulation results show good agreement with the data and demonstrate that the detector calibration and performance are well understood.

\section{Conclusion}

The KOTO experiment at J-PARC seeks to achieve the first observation of the $K_{L}^{0} \rightarrow \pi^{0} v \bar{v}$ decay. Almost all the detector subsystems have been fabricated and installed in December 2012. An engineering run was performed from December 2012 to January 2013. The calibration method to each detector subsystem was established, and data were taken to check the detector performance. Preliminary results presented in this paper demonstrate how we understand of the detector; more detailed analysis works are in progress. The KOTO experiment is ready to start the physics run to investigate new physics in the middle of May 2013. 

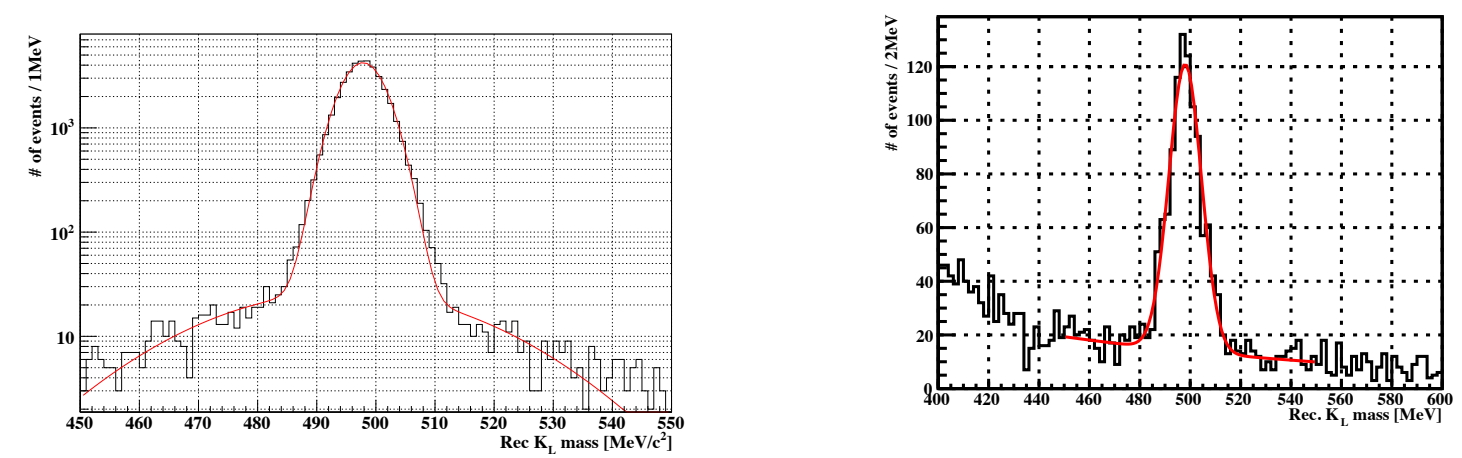

Figure 10: Reconstructed invariant mass of the $K_{L} \rightarrow 3 \pi^{0}$ events. $\begin{aligned} & \text { Figure 11: } \\ & 2 \pi^{0} \text { events. }\end{aligned}$

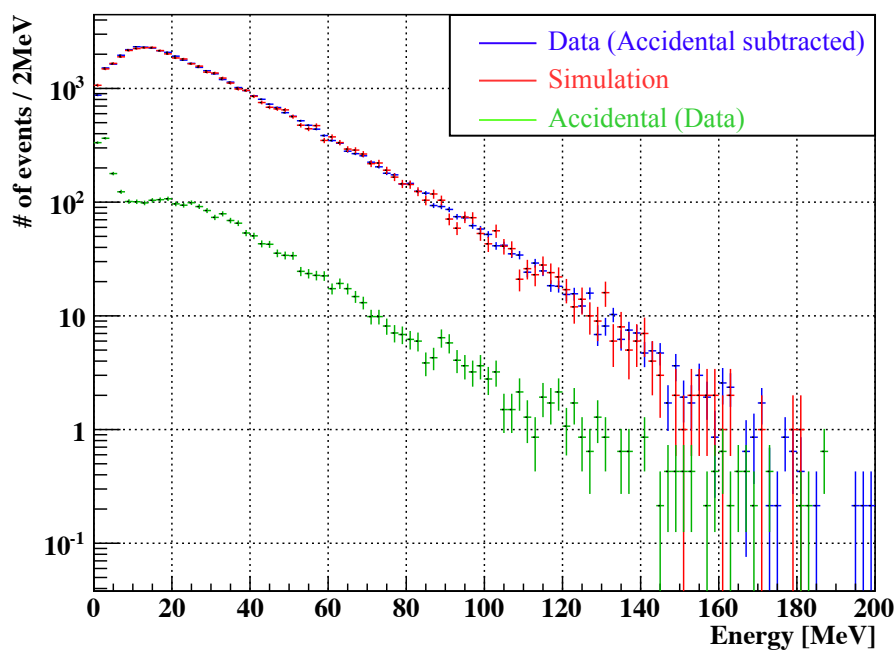

Figure 12: Energy spectrum of MB in the low-mass region of the $K_{L} \rightarrow 2 \pi^{0}$ events.

\section{References}

[1] David Tytler, et al., 2000 Phys. Scr. 200012.

[2] T. Yamanaka, et al., Prog. Theor. Exp. Phys. 2012, $02 B 006$ (2012).

[3] J. Brod, M. Gorbahn, and E. Stamou, Phys. Rev. D 83, 034030 (2011).

[4] J. K. Ann, et al., Phys. Rev. D 81, 072004 (2010).

[5] Y. Maeda, arXiv:1212.3678 (2012).

[6] Y. Tajima, et al., Nucl. Instrum. Methods A 592, 261-272 (2008). 